Kompass

Autoimmun

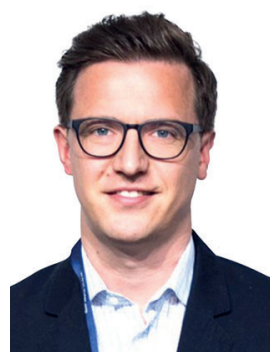

Philipp Sewerin

Koordination «AutoimmunCampus»

\title{
Immunologische Prozesse als Initiale für Kooperationen
}

Das Verständnis für immunologische Prozesse ist heute besser denn je. Gerade für junge Kolleginnen und Kollegen sind diese komplexen Zusammenhänge jedoch häufig schwierig zu erfassen und in den klinischen Alltag zu übertragen. In der aktuellen Ausgabe möchten wir daher diese Prozesse beleuchten und Ihnen einen Einblick in die neuen Erkenntnisse geben.

So wird Herr Dr. Mike Oliver Becker aus Zürich Ihnen im «Blickfang Pathogenese: Vaskulitiden» einen Einblick in bekannte und neue Erkenntnisse zu Entwicklung und Verlauf von Vaskulitiden präsentieren.

Im Anschluss möchten wir Ihnen das MalHeuR-Register aus Heidelberg vorstellen, welches unter anderem die Koinzidenz von Malignomen und entzündlich-rheumatischen Erkrankungen in verschiedenen Subregistern untersucht. Dieses Thema ist für uns auch und insbesondere im klinischen Alltag von großer Bedeutung, da uns die Fragen nach möglichen immunsuppressiven/immunmodulierenden Therapien immer häufiger auch bei bestehenden oder stattgehabten Tumorerkrankungen erreichen. Die Einführung neuer Wirkprinzipien - wie beispielsweise die der Check-Point-Inhibitoren - wird hier die Kooperation, sei es klinisch oder wissenschaftlich, sicherlich nochmals verstärken.

Zudem stellt sich die Arbeitsgemeinschaft der Jungen Rheumatologen (AGJR) innerhalb der Deutschen Gesellschaft für Rheumatologie (DGRh) vor. Mit über 200 aktiven Mitglieder ist die AGJR heute ein wichtiger Teil der DGRh und unterstützt insbesondere die Arbeit in den Themenfeldern Education, Innovation und Networking. Hier konnten bereits erste eigene Projekte, gerade im Bereich der Digitalisierung, publiziert werden.

Wir hoffen, dass Ihnen unsere aktuelle Ausgabe einen Mehrwert liefert und freuen uns sehr über Ihre Rückmeldungen.

Ich wünsche Ihnen viel Freude beim Lesen! 


\section{Blickfang Pathogenese}

\section{Vaskulitiden FOKUS}

Die Abbildung gibt einen schematischen Überblick zur Pathogenese der Riesenzellarteritis und mögliche pharmakologische Intervention durch Biologika. Vaskuläre dendritische Zellen (vasDC) aktivieren T-Helferzellen (Th1, Th17), indem sie Antigene im Kontext von Humanen Leukozyten-Antigenen (HLA) präsentieren. Abatacept kann an CD80/86 binden und blockiert somit das Co-simulierende Signal für die Aktivierung der T-Helferzelle. Sowohl B-Lym- phozyten (B cell) als auch vasDCs sezernieren Interleukin-6 (IL-6), welches Th17-Helferzellen stimuliert. Sirukumab blockiert lösliches IL-6, Tocilizumab und Sarilumab beeinflussen die Bindung von IL-6 am Rezeptor. VasDC und Antigen-präsentierende Zellen sezernieren zusätzlich IL-12 und IL-23 und stimulieren damit Th1 und Th17. Ustekinumab bindet an eine Untereinheit von Interleukinen (IL-12 und IL-23) und blockiert deren Wirkung.

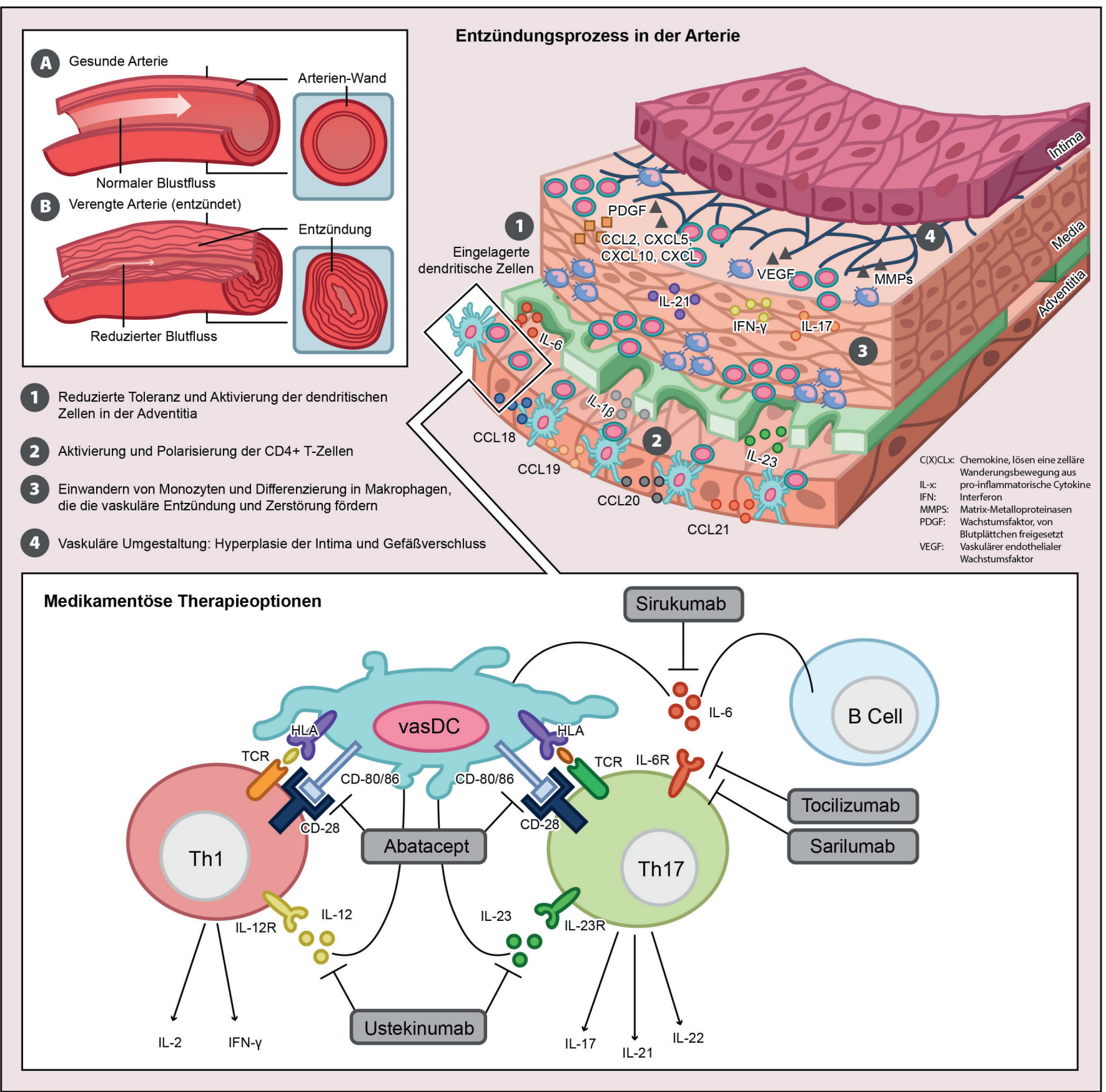

Grafik kombiniert und modifiziert aus

(1) Hid Cadena R et al. Front Immunol. DOI 10.3389/fimmu.2018.00315.

(2) Koster and Warrington. BMC Rheumatology. DOI 10.1186/s41927-017-0004-5

(3) https://www.nhlbi.nih.gov/health-topics/vasculitis
Autor: Dr. Mike Oliver Becker Klinik für Rheumatologie, Universitätsspital Zürich 


\section{Aus der Praxis \\ Das Heidelberger MalheuR-Register: Maligne und rheumatische Erkrankungen - zwei Extreme des fehlgesteuerten Immunsystems}

Die internistische Rheumatologie umfasst etwa 400 entzündlichrheumatische Erkrankungen autoimmuner oder autoinflammatorischer Genese, die etwa 2\% der erwachsenen Bevölkerung in Deutschland betreffen. Wie kaum ein anderer Fachbereich hat die Rheumatologie in den letzten Jahren von wissenschaftlicher Forschung und der Zulassung innovativer Medikamente profitiert. Dadurch hat sich nicht nur die Funktionalität der Patienten erheblich verbessert (weniger Arbeitsunfähigkeit/Erwerbsminderung und körperliche Behinderung/Pflegebedürftigkeit), sondern ist auch die Lebenserwartung signifikant gestiegen. Sowohl die rheumatische Grunderkrankung als auch immunmodulatorische bzw. -suppressive Therapien stellen jedoch Risikofaktoren für verschiedene Begleit- und Folgeerkrankungen dar. Besondere Relevanz haben in diesem Zusammenhang maligne Erkrankungen, deren Inzidenzund Prävalenzraten in der Allgemeinbevölkerung insbesondere aufgrund der demografischen Entwicklung stetig zunehmen. Gleichzeitig ist auch im Fachgebiet der Onkologie durch klinische und experimentelle Fortschritte ein Rückgang der Malignom-assoziierten Sterblichkeitsrate zu verzeichnen, sodass die Anzahl an Langzeitüberlebenden nach Malignomdiagnose zunimmt.

Aufgrund der zunehmenden Prävalenz maligner und entzündlichrheumatischer Erkrankungen ist die Wahrscheinlichkeit für eine Koinzidenz beider Krankheitsentitäten in einem Individuum entsprechend höher. Allerdings sind die Zusammenhänge und Wechselwirkungen auf klinischer und molekulargenetischer Ebene bislang nur in Ansätzen erforscht. Der Mangel an einerseits verlässlichen Daten zum krankheits- und therapieassoziierten Malignom(rezidiv)Risiko bei bestimmten entzündlich-rheumatischen Erkrankungen und andererseits an standardisierten Handlungsempfehlungen bei Koinzidenz beider Krankheitsentitäten verunsichert im klinischen Alltag sowohl die Patienten als auch behandelnde Ärzte. In der Folge werden rheumatische Symptome bei Patienten mit Malignomen regelhaft untertherapiert, unter der Vorstellung, mögliche negative Effekte auf die maligne Erkrankung (Tumorprogress bzw. Verlust des Ansprechens auf Tumortherapie etc.) zu vermeiden.

Um diese Lücken zu schließen, wurde das MalheuR-Projekt (Akronym aus malignen und rheumatischen Erkrankungen) als Registerstudie von der Sektion Rheumatologie des Universitätsklinikums Heidelberg 2018 initiiert. Die Koinzidenz von entzündlich-rheumatischen Erkrankungen und Malignomen wird dabei aus 3 verschiedenen Dimensionen in Subregistern betrachtet, die im Weiteren näher dargestellt werden

\section{Rheumatologische Patienten mit Malignom (RheuMal-Register)} Wenn Patienten mit entzündlich-rheumatischen Erkrankungen ein Malignom entwickeln, ist sowohl ein Zusammenhang mit der rheumatischen Grunderkrankung (z.B. erhöhtes Lymphomrisiko beim Sjögren-Syndrom) als auch mit der durchgeführten Therapie zu diskutieren (z.B. erhöhte Risiken für Urothelkarzinome nach Cyclophosphamid, für Hautmalignome nach Azathioprin oder nach therapeu- tischer UV-Bestrahlung (PUVA) der Psoriasis bei Psoriasis-Arthritis). Für viele medikamentöse Therapien sind die Zusammenhänge nicht endgültig geklärt. Die Risikobeurteilung wird zusätzlich durch teilweise erst kurze Zulassungsdauer und damit geringe Erfahrung einerseits sowie kombinierte bzw. sequentielle Anwendung verschiedener Basistherapeutika andererseits erschwert.

Entsprechend groß ist die Verunsicherung bezüglich des Malignomrisikos, insbesondere bei Therapien, die an zentralen Schaltstellen des Immunsystems eingreifen. Die landesweiten Register leisten zwar einen wesentlichen Beitrag bei der Langzeitbeobachtung und Erfassung seltener Risiken bestimmter Therapien, jedoch fehlen bisher größtenteils krankheits- und therapieübergreifende Korrelationen der Patientencharakteristika, der rheumatischen und malignen Diagnosen, der Krankheitsverläufe und -Outcomes und ein Vergleich zur rheumatisch nicht vorbelasteten Allgemeinbevölkerung.

Hier setzt das RheuMal-Register an, das bereits erste Hinweise erbringen konnte, dass Patienten mit entzündlich-rheumatischen Erkrankungen früher an bestimmten Malignomen erkranken können als die gesunde Vergleichspopulation. Zusätzlich soll die Datenlage zum klinischen Management der entzündlich-rheumatischen Erkrankung und dem Rezidivrisiko nach der Diagnose des Malignoms gestärkt werden.

\section{Paraneoplastische Rheumatische Symptome bei Malignom- patienten (ParaRheuMa-Register)}

Unspezifische muskuloskelettale Symptome und Funktionseinschränkungen werden zumindest zeitweise bei etwa einem Viertel der Allgemeinbevölkerung beobachtet, stellen demnach häufige Ereignisse dar und werden daher regelhaft als mögliches Leitsymptom einer malignen Erkrankung nicht ernst genommen. Paraneoplastische Symptome werden infolgedessen häufig erst verzögert identifiziert, in aller Regel durch den atypischen, therapierefraktären Verlauf einer vermeintlichen entzündlich-rheumatischen Erkrankung, mit konsekutiver Diagnose der malignen Erkrankung. Charakteristisch für eine Paraneoplasie ist dabei nicht nur die zeitliche Assoziation (synchrones, allenfalls wenige Monate auseinanderliegendes Auftreten der Symptome), sondern auch die Beobachtung, dass die Symptome häufig nur durch die erfolgreiche Malignomtherapie nachhaltig positiv beeinflusst werden.

Im Rahmen des ParaRheuMa-Registers soll insbesondere erforscht werden, ob einerseits bestimmte Symptomkomplexe gehäuft paraneoplastisch auftreten und andererseits Zusammenhänge zwischen paraneoplastischen muskuloskelettalen Symptomen und bestimmten Neoplasien existieren.

\section{Therapieinduzierte Rheumatische Symptome bei Malignom- patienten (TRheuMa-Register)}

Neue Aufmerksamkeit erlangten die wechselseitigen Beziehungen zwischen entzündlich-rheumatischen und malignen Erkrankungen DOI: $10.1159 / 000505559$ 
durch das Auftreten rheumatischer Symptome als Nebenwirkungen der Immuntherapie mit Checkpoint-Inhibitoren (sogenannte «immune-related adverse events» (irAEs)). Der Wirkmechanismus dieser Substanzen besteht v.a. in der Blockierung der über Checkpoint-Rezeptoren vermittelten Escapemechanismen von Tumorzellen mit erneuter Aktivierung der physiologischen Tumorclearance durch zytotoxische T-Zellaktivität. Hierbei wird jedoch eine überschießende Immunantwort mit reduzierter Toleranz gegenüber körpereigenen Zellen ausgelöst: irAEs können alle Organsysteme betreffen und klinisch bekannten Autoimmunopathien ähneln. So können auch alle Krankheitsbilder des rheumatischen Formenkreises nachgeahmt werden, wobei Arthralgien und/oder Myalgien als milde irAEs häufig auftreten und in klinischen Studien von 10-20\% der Patienten berichtet wurden. Da häufig typische serologische (Autoantikörper) Befunde «klassischer» Erkrankungen fehlen, ergibt sich derzeit meist nur aus dem Verlauf, ob es sich um eine immunvermittelte entzündliche Nebenwirkung oder eine therapiegetriggerte Erstmanifestation einer tatsächlichen entzündlich-rheumatischen Erkrankung handelt. Obgleich rheumatische irAEs selten vital gefährdend sind, können sie dennoch erhebliche Schmerzen und Funktions-, bzw. Mobilitätseinschränkungen verursachen, die entsprechender Behandlung bedürfen. Besondere Relevanz haben die rheumatischen irAEs dahingehend, dass betroffene Patienten ein besseres Tumoransprechen und Langzeitüberleben aufweisen. Dieser Vorteil soll möglichst nicht durch eine immunmodulierende/ -supprimierende Therapie beeinträchtigt werden.

Die Ziele des TRheuMa-Registers sind, aus der rheumatologischen Perspektive erstmals prospektiv eine systematische Übersicht zu Häufigkeit, Art und Verlauf muskuloskelettaler Nebenwirkungen unter antineoplastischen Therapien zu erstellen. Besonderer Fokus sind hierbei zwar die irAEs unter Checkpoint-Inhibition, prinzipiell können aber auch andere antineoplastische Therapien wie beispielsweise Aromatasehemmer bei Malignompatienten rheumatische Symptome verursachen.

\section{Forschungsagenda}

Die weitere Erforschung beider Krankheitsentitäten im Rahmen des MalheuR-Registers bietet die einzigartige Möglichkeit, zwei Extreme des fehlgeleiteten Immunsystems auch auf der grundlagenwissenschaftlichen Ebene näher zu charakterisieren. Die NeoplasieEntstehung mit verminderter Tumorsurveillance und pathologisch reduzierter Immunantwort auf der einen Seite sowie die Autoimmunopathie-Entstehung mit autoaggressiver Immunzellaktivität als Gegenpol bieten wertvolle Ansatzpunkte für weitere Forschungsarbeit: Durch Verknüpfung des Registers mit bestehenden Biobank-Projekten können Hinweise auf Pathogenese beider Entitäten gewonnen und potenziell neue Biomarker und Therapietargets identifiziert werden.

\section{Fazit}

Die bisher nicht dagewesene Betrachtung der Zusammenhänge und Wechselwirkungen entzündlich-rheumatischer und maligner Erkrankungen aus 3 Blickwinkeln zeichnet das MalheuR-Register aus. Durch die Erforschung der Schnittstellen zwischen beiden Krankheitsentitäten basierend auf demographischen Daten, Latenzzeiterfassung der Charakteristika, therapeutischen Managements und Outcomes sowie der Korrelationen spezifischer Diagnosen und Therapien kann ein Wissenszuwachs generiert werden, der betroffenen Patienten auf mehreren Ebenen zugutekommt und bestehende Unsicherheiten im klinischen Alltag mindern kann.

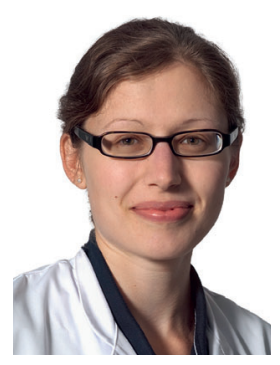

Dr. Karolina Benesova

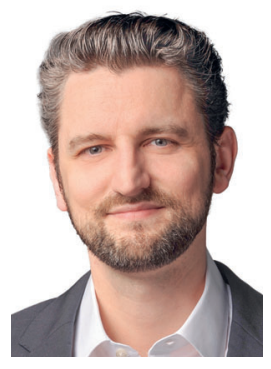

Dr. Jan Leipe
Autoren

Dr. Karolina Benesova, Universitätsklinik Heidelberg, Innere Medizin V, Sektion Rheumatologie, Heidelberg; Dr. Jan Leipe, Universitätsklinik Mannheim, V. Medizinische Klinik, Sektion Rheumatologie, Mannheim

\section{Literatur}

1 Benesova K, Lorenz H-M, Leipe J, et al. How I treat cancer: treatment of rheumatological side effects of immunotherapy. ESMO Open 2019;4.

2 Buder-Bakhaya K, Benesova K, Schulz C, et al. Characterization of arthralgia induced by PD-1 antibody treatment in patients with metastasized cutaneous malignancies. Cancer Immunol Immunother 2018;67:175-82.

3 Leipe J, Christ LA, Arnoldi AP, et al. Characteristics and treatment of new-onset arthritis after checkpoint inhibitor therapy. RMD Open 2018;4:e000714.

4 Zink A, Albrecht K, Wie häufig sind muskuloskeletale Erkrankungen in Deutschland? Z Rheumatol 2016, 75:346-353. 


\section{Arbeitsgemeinschaft Junge Rheumatologie (AGJR) - rheumadocs stellt sich vor}

Fortbilden - Erneuern - Vernetzen, das sind die drei wesentlichen Ziele der Arbeitsgemeinschaft Junge Rheumatologie - rheumadocs (AGJR). Hervorgegangen aus dem Verein rheumadocs e.V., bildet die AGJR seit Anfang des Jahres 2019 eine eigene Arbeitsgemeinschaft in der Deutschen Gesellschaft für Rheumatologie e.V. (DGRh). Ihr gehören junge Medizinerinnen und Mediziner im Alter unter 40 Jahren an, die sich für das Fach Rheumatologie begeistern und Mitglieder in der DGRh sind. Die AGJR sucht stetig Zuwachs, um möglichst alle jungen Kolleginnen und Kollegen aus der Rheumatologie miteinander zu vernetzen. Auf diese Weise kann eine interaktive Plattform für regen Austausch, ganz im Sinne unserer drei Ziele Education, Innovation, Networking angeboten werden.

\section{Die drei Ziele der AGJR}

Education: Beeinflussung und Unterstützung der Aus-, Weiterund Fortbildung junger Kolleginnen und Kollegen in der Rheu matologie auf dem Weg zum Facharzt und danach.

Innovation: Zukunftsorientierte und digitale Projekte in der Medizin und den Medien.

Networking: Vernetzen aller jungen Ärztinnen und Ärzte in der Rheumatologie.

Gegründet wurden die rheumadocs e.V. im Jahr 2011 als gemeinnütziger Verein. Seit 2012 vereint rheumadocs mittels Internetplattform junge rheumatologisch interessierte Akademiker [1]. Seit Januar 2019 sind wir als AGJR - rheumadocs als Teil der DGRh aktiv. Damit steht dem Sprecher der AGJR eine Drittel-Stimme bei Vorstandsbeschlüssen der Fachgesellschaft zu. Die Arbeitsgemeinschaft wird in der DGRh derzeit von unserem Sprecher PD Dr. Philipp Sewerin (Düsseldorf), seinem Co-Sprecher Dr. Arnd Kleyer (Erlangen) sowie den drei Ausschussmitgliedern Dr. Martin Krusche (Berlin), Dr. Johanna Mucke (Düsseldorf) und Dr. Diana Vossen (Meerbusch) repräsentiert. Aktuell sind 201 Mitglieder in der AGJR registriert und engagieren sich aktiv in der Gestaltung und Umsetzung unserer Projekte. Wir arbeiten in enger Kooperation mit anderen nationalen und internationalen Organisationen zusammen wie z.B. EMEUNET (Vertretung der Jungrheumatologen der European League Against Rheumatism (EULAR)). Verschiedene Firmen fördern unsere Arbeit im Rahmen einer korporativen Mitgliedschaft.

Die Förderung der Aus- und Weiterbildung (Education) setzt sich neben den internen Fortbildungen u.a. auch aus Stipendien für Facharztvorbereitungskurse und Kongressstipendien zusammen. Ebenso engagieren wir uns in zahlreichen Kommissionen der DGRh und sind so aktiv an der Gestaltung der Weiterbildungsordnung in der Kommission Fort- und Weiterbildung sowie an der Umsetzung und Weiterentwicklung digitaler Projekte in der Kommission digitale Rheumatologie der DGRh beteiligt. Weiterhin organisiert die AGJR eigene Fortbildungen, z.B. ein Speakerstraining zum Erlernen der Präsentation wissenschaftlicher Arbeiten.

Neben dem Engagement in der AG selbst und in Kommissionen konnten wir bereits eigene wissenschaftliche Projekte verfolgen (In- novation) und veröffentlichen, zuletzt auch mit einer internationalen Publikation zur Bewertung von Apps in der Rheumatologie [2]. Bereits bei rheumadocs e.V. war die Vernetzung (Networking) - sei es aus wissenschaftlichem oder klinischem Interesse - eine der wichtigsten Aufgaben des Vereins. Auch aus diesem Grund findet zweimal jährlich eine Klausurtagung statt, um die nächsten Ziele und Projekte der AGJR zu planen und zu koordinieren. Jeweils im Januar organisieren wir das Fellowmeeting, das neben dem Networking auch der dem Ausbildungsstand angemessenen Weiterund Fortbildung sowie der Integration neuer Mitglieder dient. Inhaltlich wird über wissenschaftliche Themen, beruflichen Zukunftsperspektivenundu.a.Weiter-undFortbildungsmöglichkeiten diskutiert. Neben fachlichem Austausch und lebhafter Diskussion werden im Rahmen des Fellowmeetings vor allem praktische Fertigkeiten wie beispielsweise das Erlernen der Grundlagen in der Arthrosonografie, das Erheben krankheitsbezogener Scores bei Patienten mit rheumatologischen Erkrankungen oder die praktische Vertiefung der Labordiagnostik vermittelt. Auch die Vernetzung mit anderen Fachdisziplinen und das «voneinander Lernen» sind wichtige Bausteine unserer Veranstaltungen. So stellte auf einem unserer Fellowmeetings eine Vertreterin der jungen Neurologen die Strukturen ihres Gremiums vor, Anregungen wurden aufgenommen und im Rahmen eines fachübergreifenden Dialogs diskutiert. Wir sind auf weiteren interdisziplinären Austausch mit angrenzenden Fachgebieten der Rheumatologie gespannt.

Zusammenfassend möchte die AGJR nun als fester Bestandteil der DGRh aktiv die Zukunft in der Rheumatologie mitgestalten - sei es in konkreten Projekten, in Kommissionen oder in innovativen Projekten und neuen Konzepten. Es ist uns ein wichtiges Anliegen, die Arbeit in der Rheumatologie selbst sowie die Bedingungen wissenschaftlichen Arbeitens kontinuierlich zu verbessern und hierbei die Interessen der jungen Kolleginnen und Kollegen zu vertreten.

\section{Literatur \\ 1 Junge Rheumatologie - rheumadocs: https://rheumadocs.de \\ 2 Knitza J, Tascilar K, Messner EM, et al.: German mobile apps in rheumatology: review and analysis using the Mobile} Application rating Scale (MARS). JMIR Mhealth Uhealth 2019;7:e14991.

Autoren: Dr. Diana Vossen (AGJR; Rheinisches Rheumazentrum Meerbusch, St. Elisabethkrankenhaus, Meerbusch), Dr. Isabelle Geffken (AGJR; Rheinisches Rheumazentrum Meerbusch, St. Elisabethkrankenhaus, Meerbusch), Dr. Johanna Mucke (AGJR; Poliklinik, Funktionsbereich und Hiller-Forschungszentrum für Rheumatologie, Universitätskliniken Düsseldorf, Heinrich-Heine-Universität Düsseldorf, Poliklinik für Rheumatologie, Düsseldorf), Dr. Martin Krusche (AGJR; Medizinische Klinik mit Schwerpunkt Rheumatologie und Klinische Immunologie, Charité Universitätsmedizin, Berlin), Dr. Arnd Kleyer (AGJR; Medizinische Klinik 3 - Rheumatologie und Immunologie, Universitätsklinikum Erlangen, Friedrich-Alexander-Universität Erlangen-Nürnberg, Erlangen), PD Dr. Philipp Sewerin (AGJR; Poliklinik, Funktionsbereich und Hiller-Forschungszentrum für Rheumatologie, Universitätskliniken Düsseldorf, Heinrich-Heine-Universität Düsseldorf, Poliklinik für Rheumatologie, Düsseldorf) 